\title{
A MATLAB APP TO INTRODUCE CHEMICAL ENGINEERING DESIGN CONCEPTS to Engineering Freshmen through a Pharmaceutical Dosing Case Study
}

\author{
Carley V. Eastep, Grace K. Harrell, Alexandra N. McPeak, and Ashlee N. Ford Versypt \\ Oklahoma State University • Stillwater, OK 74078
}

$\mathrm{H}$ ands-on design experiences to introduce first year students to chemical engineering are limited. ${ }^{[1]}$ The College of Engineering, Architecture and Technology (CEAT) Summer Bridge Program at Oklahoma State University is a three-week camp designed to acclimate first year students to college life while providing calculus and physics preparatory short courses and design experiences from multiple disciplines in CEAT. ${ }^{[2]}$ Since firstyear students enjoy and value hands-on experiences ${ }^{[2]}$ we have developed a hands-on design module on the topic of pharmaceutical dosing for the chemical engineering portion of the Summer Bridge Program. We have taught the design module in nine sessions that were each two hours per day over three days during the 2015 - 2018 offerings of the Summer Bridge Program. The detailed lesson plan for using the design module to introduce mass balance and design concepts and an overview of the initial version of the MATLAB app were discussed in our previous ASEE conference proceedings paper. ${ }^{[2]}$ In the present work, we discuss a redesigned MATLAB app (Figure 1) that has been expanded from two drugs and four cases to five drugs and eight cases. The new version of the MATLAB app is available as SB17CHE.mlappinstall on our GitHub repository. ${ }^{[3]}$ Any future updates to the software will be released to the same archive. Additionally, we include a new section focused on our experiences in teaching chemical engineering design concepts using the MATLAB app and in responding to typical student questions. Our aim is to provide a tutorial for other educators interested in using the MATLAB app in their classroom or outreach activities related to chemical engineering design. For those interested in creating similar computational instructional tools, we have published details regarding the software implementation of the app and its documentation. ${ }^{[4]}$

\section{METHODS}

Our goals for the MATLAB app and the associated activities that constitute the design module are to engage students through an engineering design project and to motivate them to major in chemical engineering. We selected a pharmaceutical example of chemical engineering design because most students have prior experience with taking medications and because of the authors' research interests in this area. The MATLAB app simulates the pharmaceutical regulation of blood pressure by controlling levels of the hormone angio-

Carley V. Eastep is a chemical engineering undergraduate student at Oklahoma State University (OSU) graduating in May 2019. She updated the MATLAB app significantly and facilitated the CEAT Summer Bridge Program sessions in 2017 and 2018.

Grace K. Harrell is pursuing a software engineering career at Quorum Business Solutions in Dallas, TX. She earned a B.S. degree in chemical engineering from OSU in May 2016. She helped facilitate the CEAT Summer Bridge Program chemical engineering design session in 2015.

Alexandra (Alex) N. McPeak is employed by International Paper at their Valliant, OK mill. She earned a B.S. degree in chemical engineering from OSU in May 2016. Her current role is in the Manufacturing Excellence group as a Process Engineer for paper machines. She helped facilitate the CEAT Summer Bridge Program chemical engineering design session in 2015.

Ashlee N. Ford Versypt is an assistant professor in the School of Chemical Engineering at OSU. She earned a B.S. from the University of Oklahoma and an M.S. and a Ph.D. from the University of Illinois at Urbana-Champaign all in chemical engineering. She was awarded the Department of Energy Computational Science Graduate Fellowship and the National Science Foundation Graduate Research Fellowship. She was recognized as the Frederick A. Howes Scholar in Computational Science and earned the Certificate in Foundations of Teaching from the UIUC Center for Teaching Excellence. In 2012 - 2014, she was a postdoctoral research associate at the Massachusetts Institute of Technology. She leads a computational research group focused on systems biomedicine and pharmaceutics. She was named as one of AlChE's 35 Under 35 for 2017. She is currently a Director of the Chemical Engineering Division of ASEE. She received the NSF Career Award in 2019.

(C) Copyright ChE Division of ASEE 2019 
tensin II via a class of pharmaceuticals called angiotensin converting enzyme (ACE) inhibitors. For an in-depth description of the mathematical model that underlies the MATLAB app, see our technical paper. ${ }^{[5]}$ The first version of the MATLAB app was parameterized for two ACE inhibitor drugs benazepril and cilazapril for patients with both normal and impaired kidney function (KF). We have expanded model parameters to also cover lisinopril for normal and impaired $\mathrm{KF}^{[6]}$ delapril for normal $\mathrm{KF},{ }^{[7]}$ and ramipril for impaired $\mathrm{KF}^{[8,9]}$ based on available data in the literature. Each drug and KF case has a distinct set of model parameters for the pharmacokinetics and pharmacodynamics of processes including absorption into the bloodstream from the digestive tract, elimination from the body, and interaction of the drug with the production of the hormone. The elimination rate constant for a given drug is always smaller in the impaired $\mathrm{KF}$ case than in the corresponding normal KF case.

To run the MATLAB app, first download the MATLAB app install file SB17CHE.mlappinstall from our GitHub

TABLE 1

Design cases. drug 1: benazepril, drug 2: cilazapril, drug 3: lisinopril, drug 4: delapril, drug 5: ramipril

\section{Case Drug Kidney Function}

\begin{tabular}{|llc|}
\hline 1 & 1 & Normal \\
\hline 2 & 2 & Normal \\
\hline 3 & 3 & Normal \\
\hline 4 & 4 & Normal \\
\hline 5 & 1 & Impaired \\
\hline 6 & 2 & Impaired \\
\hline 7 & 3 & Impaired \\
\hline 8 & 5 & Impaired \\
\hline
\end{tabular}
ous combinations of ACE inhibitor drugs on virtual patients with normal or impaired KF. The MATLAB app allows for eight possible design cases (Table 1).

In the chemical engineering design session of the Summer Bridge Program, students work in small teams to design the

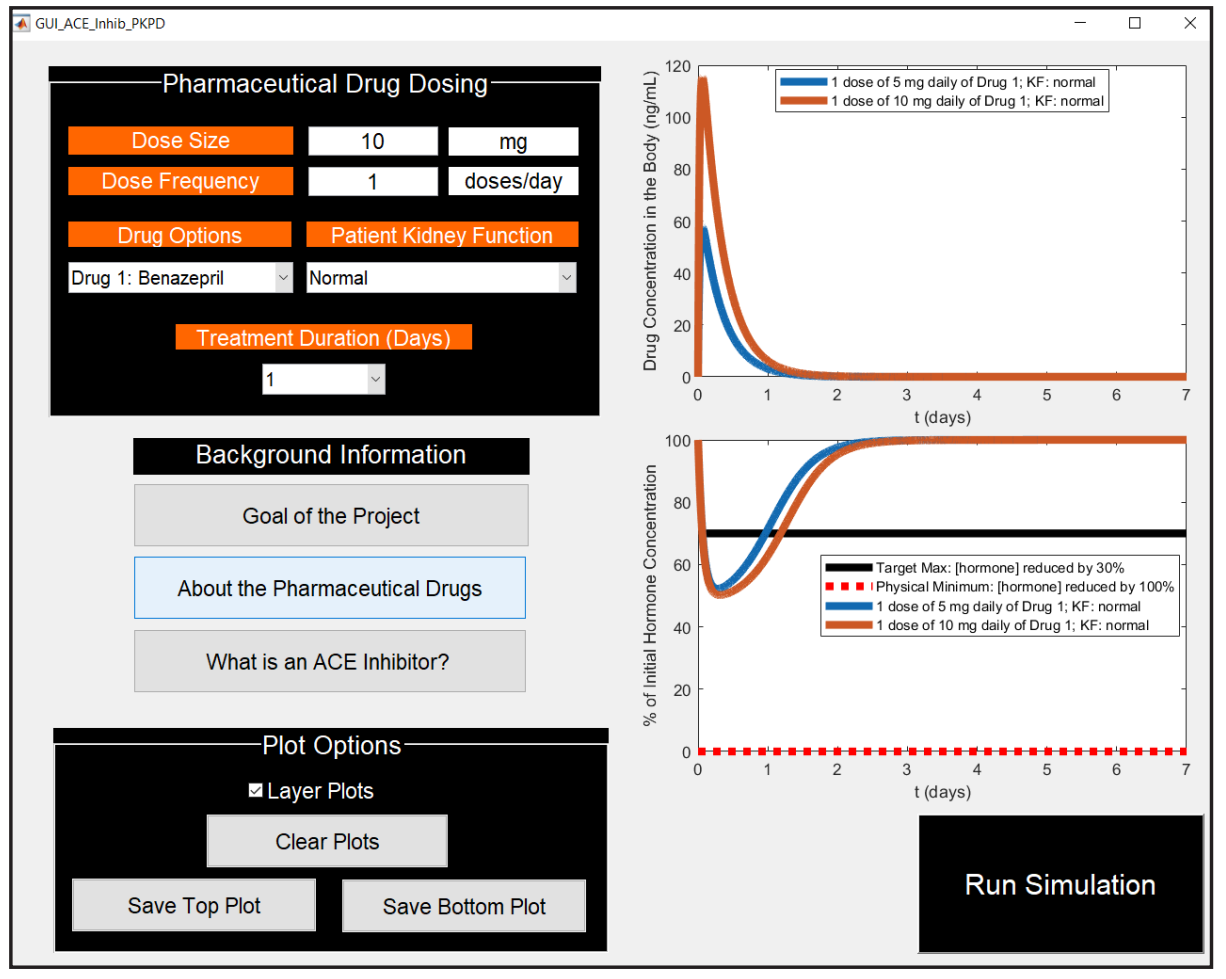

Figure 1. MATLAB graphical user interface for the drug dosing app for ACE inhibitor pharmaceuticals for normal and impaired kidney function..$^{[2]}$ Reproduced with permission of ASEE, copyright 2017.

dosing schedule for one or more assigned design cases using the MATLAB app. We discuss the TeachEngineering digital library's outline of the engineering design process ${ }^{[10]}$ and apply it to our topic in a series of classroom activities (Table 2 ). At the end of the design cycle, student teams create and present a one-slide presentation (Figure 2) to convey their results to their peers in five minutes or less.

\section{EXPERIENCES}

Students have enjoyed designing pharmaceutical dosing schedules using the MATLAB app. To assess interest in chemical engineering, we have asked the participants over age 18 from the Summer Bridge Program to complete a short, anonymous, and voluntary feedback survey at the conclusion of the sessions. The average of 52 responses to the prompt "level of knowledge of/interest in chemical engineering before the project" was 2.51/5. This average improved to $3.38 / 5$ for the corresponding prompt after the project. The project has not yet been incorporated into a formal course, so no classroom research measuring impacts on specific student learning outcomes has been conducted.

After the 2017 Summer Bridge Program and the revisions to the MATLAB app, ${ }^{[3]}$ we created a teaching guide to make the MATLAB app easier to utilize in classrooms with engineering educators who may not be familiar with the subject matter. During the sessions, we assign each team to compare 
three of the eight cases (Table 1). We strategically assign the cases so that each group encounters the results of both kidney functions with the same drug and another case with a different drug, allowing for exploration of the topics amongst each team and the whole class. Figures $3-7$ provide sample solutions for each of the five drugs that can be simulated in the MATLAB app. This guide includes suggested questions to ask students while they are testing, evaluating, and redesigning using the MATLAB app and a list of frequently asked questions along with our sample answers for each question. At the end of this section, we reflect on using the MATLAB app in different educational contexts.

Questions to ask students to guide their inquiry (followed by our sample answers)

1. How are you choosing to optimize this scenario? Are you optimizing differently depending on the case or does your target remain constant?

Optimization is the target of this exercise. Students may have vary-

\begin{tabular}{|c|c|}
\hline \multicolumn{2}{|c|}{$\begin{array}{c}\text { TABLE } 2 \\
\text { Engineering design process }{ }^{[10]} \text { mapped to classroom activities for the chemical engineering design project }\end{array}$} \\
\hline Engineering Design Process Steps ${ }^{[10]}$ & Classroom Activities for Design Project \\
\hline Identify the need and constraints & $\begin{array}{l}\text { - Introduce the problem statement: } \\
\text { "Design the best dose size and dosing } \\
\text { frequency of pharmaceuticals (raw } \\
\text { materials) that effectively reduce high } \\
\text { blood pressure (the desired product)" } \\
\text { - Brainstorm tradeoffs to be considered in } \\
\text { design of a dosing schedule (e.g., } \\
\text { convenience, efficacy, pill size, cost) }\end{array}$ \\
\hline Research the problem & $\begin{array}{l}\text { - Read websites on symptoms of high blood } \\
\text { pressure and pharmaceutical treatments } \\
\text { using ACE inhibitors }\end{array}$ \\
\hline Develop possible solutions & $\begin{array}{l}- \text { Share findings from the websites } \\
\text { - Respond to instructor questions such as: } \\
\text { How do ACE inhibitors work to } \\
\text { reduce blood pressure? } \\
\circ \quad \text { What would you expect to happen if a } \\
\text { drug dose is increased? } \\
\circ \quad \text { What would happen if a drug dose was } \\
\text { too high or too low? } \\
\text { What would you expect to happen if a } \\
\text { drug was dosed more frequently? }\end{array}$ \\
\hline Select a promising solution & - $\quad$ Get assigned design cases (Table 1) \\
\hline Build a prototype & $\begin{array}{l}\text { - Use the MATLAB app to specify the } \\
\text { design case and trial input conditions for } \\
\text { the dose size and frequency }\end{array}$ \\
\hline Test and evaluate the prototype & $\begin{array}{l}\text { - Run the simulation using the MATLAB } \\
\text { app } \\
\text { - Analyze the plots } \\
\text { Determine how the results compare to } \\
\text { expectations and how they align with } \\
\text { design criteria or tradeoffs }\end{array}$ \\
\hline Improve and redesign & $\begin{array}{l}\text { - } \\
\text { - } \text { Retest until a design is identified that best } \\
\text { meets the student-defined design } \\
\text { constraints }\end{array}$ \\
\hline
\end{tabular}

ing answers due to different opinions. Some optimization goals that we have seen are: keeping the hormone curve as flat as possible to minimize variation in hormonal levels, keeping the drug concentration low, keeping the dosing frequency low, staying close to the target maximum, staying away from the target maximum either at the middle of the target range or close to zero, and getting below the target maximum as quickly as possible. None of these interpretations are wrong, and the variance in the answers prompt discussion when the groups compare answers. A discussion can follow about needing more information about design criteria standards for pharmaceutical dosing.

2. Do you see any differences between two drugs with the same kidney function? If so, what does this mean to you?

The students should notice that some drugs need a higher dosage to fall below the target maximum and that some drugs leave the body faster than others. Students should be guided to connect these results with differences in the 


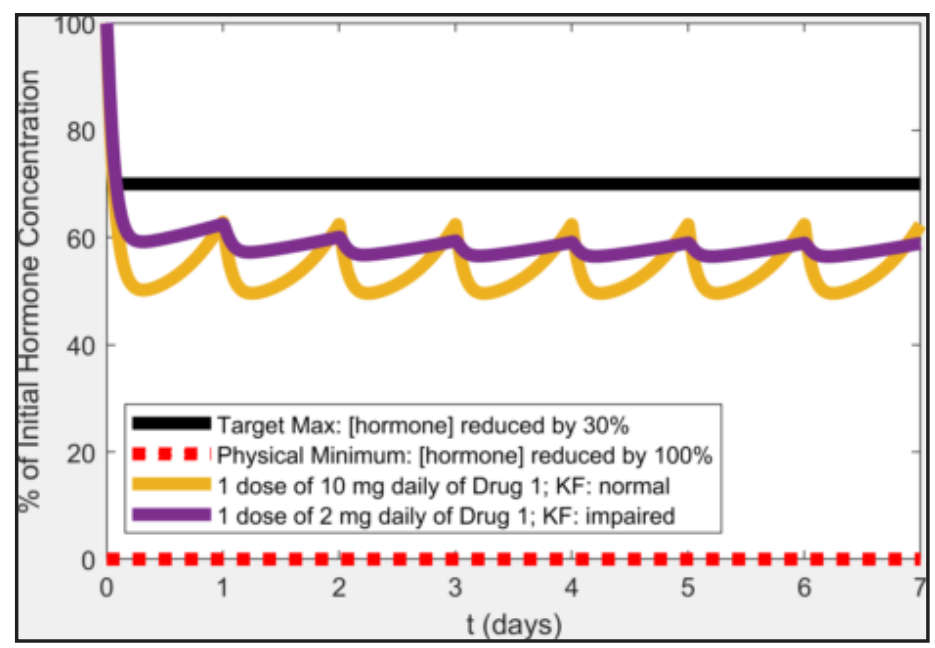

Figure 3. Possible solutions for benazepril with normal and impaired kidney function.

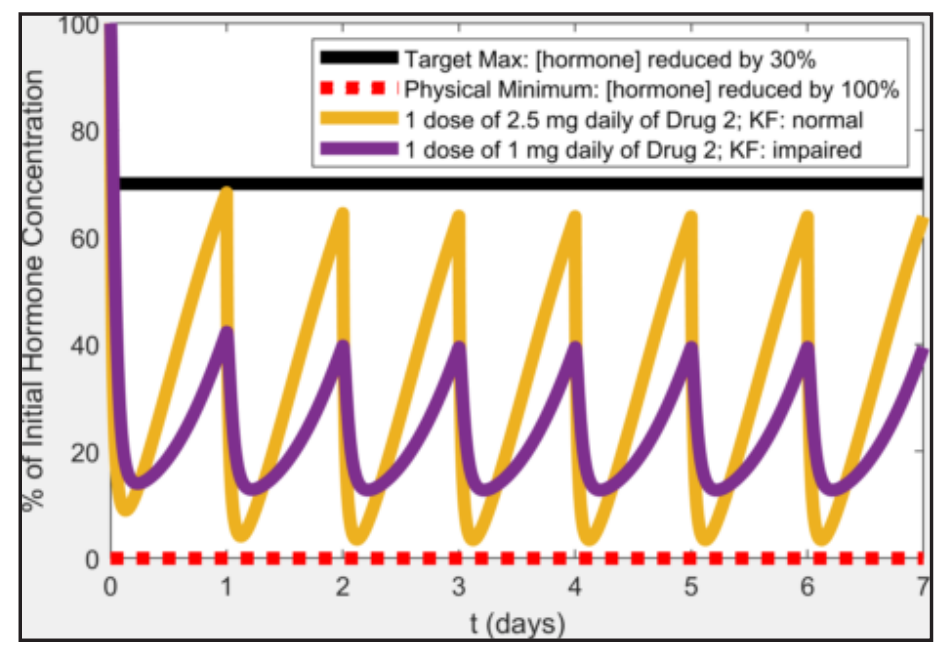

Figure 4. Possible solutions for cilazapril with normal and impaired kidney function.

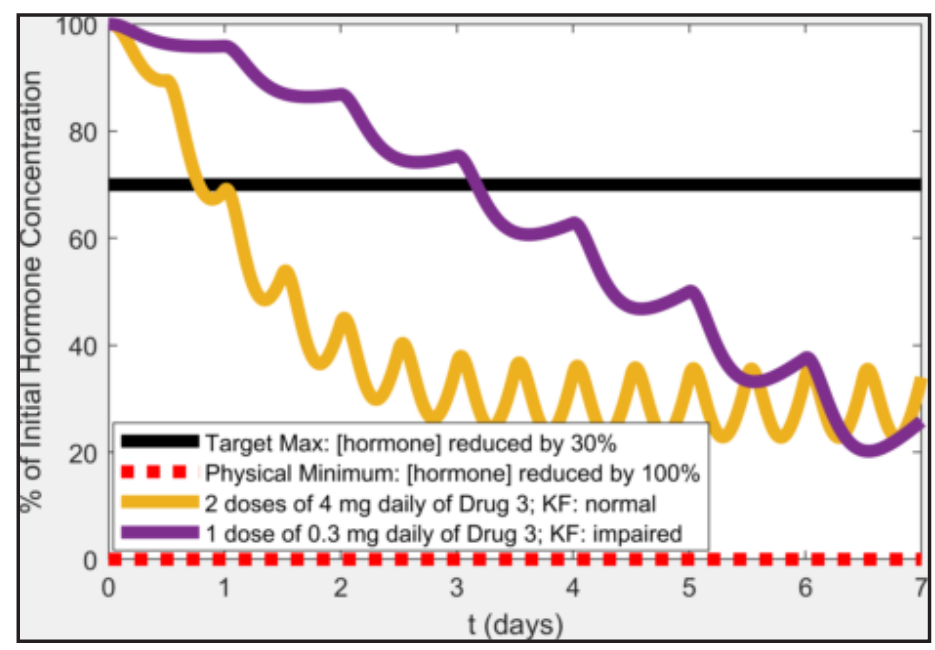

Figure 5. Possible solutions for lisinopril with normal and impaired kidney function. chemical composition that cause the drugs (a) to be processed at various rates during absorption from the digestive system into the blood stream, metabolism, and excretion from the body and (b) to have different impacts on physiological process in the body giving different drug efficacies.

\section{Do you see any differences between two kidney function with the same drug? Is so, what does this mean to you?}

If students are introduced to the idea that amounts of drug in the bloodstream can be described using mass balances ${ }^{[2]}$ they can make informed explanations of differences in the results due to kidney function. They may suggest changes to the excretion rate through the kidneys. The impaired kidney function cases require lower dosages and dosing frequencies compared to normal kidney function because the impaired kidneys do not filter out the drugs as fast as normal kidneys, leading to the drugs accumulating and staying longer in the body.

4. Based on your observations of the different drugs, would you choose to treat patients with different kidney functions with the same drug?

This question aims to facilitate a deeper understanding of the relationship between the hormone and kidney function. Using the more potent drug for a patient with normal kidney function enables a lower dosage to be used, while the impaired case still has a reasonable dose size, minimizing side effects.

5. What are some other factors that could affect the drug concentration that this model does not take into account?

This model allows the students to manipulate many variables, but it could be even more accurate if other things also could be changed. These include varying dose sizes throughout treatment, weight of the patient, physical fitness of the patient, gender of the patient, interactions with other another drugs, and patient-specific physiological or pharmacological parameters.

6. How convenient is the dosing frequency? Is it realistic to expect patients to take a medicine that many times a day?

This question should be asked if a student is attempting to set the dosage frequency more than three times per day. This might prompt the student to reevaluate their optimization and adjust to a frequency that a patient would comply with more easily.

7. Is it important to have some of the hormone remain in the body?

A student may try to keep the hormone concentration at zero. This question suggests to the student to recognize that it is important for some of the hormone to remain in the body to execute normal functions. 
8. Do you notice the relationship between drug concentration and hormone concentration? How do their graphs compare?

This question can be used to check if the student knows what they are working on. They should notice that the drug and hormone concentrations graphs move in opposite directions showing that the hormone levels rise (hormone lowering effects diminish) as the drug concentration decreases.

\section{Frequently asked questions}

\section{What does the line mean by target maximum?}

Several students will ask this question, but for the purpose of the open ended design project you should only reiterate that the hormone concentration has to be below the target maximum line to be effective.

\section{What is impaired kidney function?}

Impaired kidney function is when a kidney is damaged and no longer filters the blood effectively.

\section{How do I see the code behind this simulation?}

In the MATLAB apps menu, hover over the icon for the SB17CHE app. After a few seconds a window with more information will pop up. The hyperlink listed under File Location will direct to the code. More details are provided in the documentation. ${ }^{[4]}$

\section{Can I layer more than one type of drug or function on the same graph?}

Yes, the graphs can display more than one type of drug and function. Simply check the box that says "layer plots".

\section{Is it important to drop the hormone below the target maximum as quickly as possible?}

High blood pressure normally causes symptoms after a long period of time, so immediately dropping the hormone need not be the top priority.

Extensions of the MATLAB app beyond first year engineering projects

We have successfully used the MATLAB app outside of the Summer Bridge Program in other contexts involving elementary school children and their grandparents, middle school and high school students, and upper division undergraduate and graduate students. OSU has a three-day on-campus summer camp called Grandparent University where children and their grandparents spend a total of six hours in the classroom learning about a specific field. For this audience, we modified the design project described in this paper to fit within two 90-minute sessions. In the first session, we briefly introduced the design project and focused on introductory concepts appropriate for children aged 7-13. We spent the bulk of that session downloading and open-

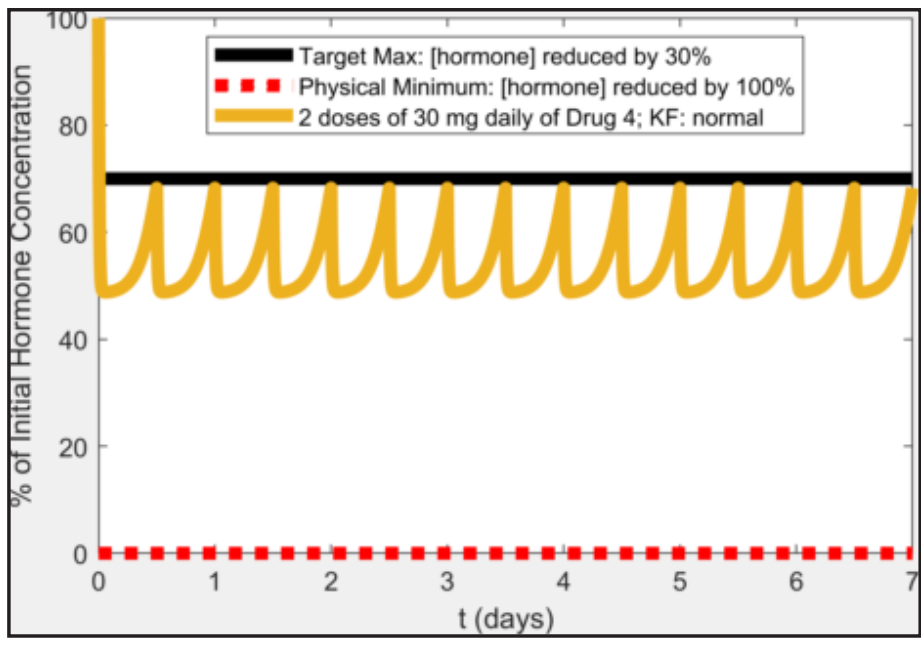

Figure 6. Possible solution for delapril with normal kidney function.

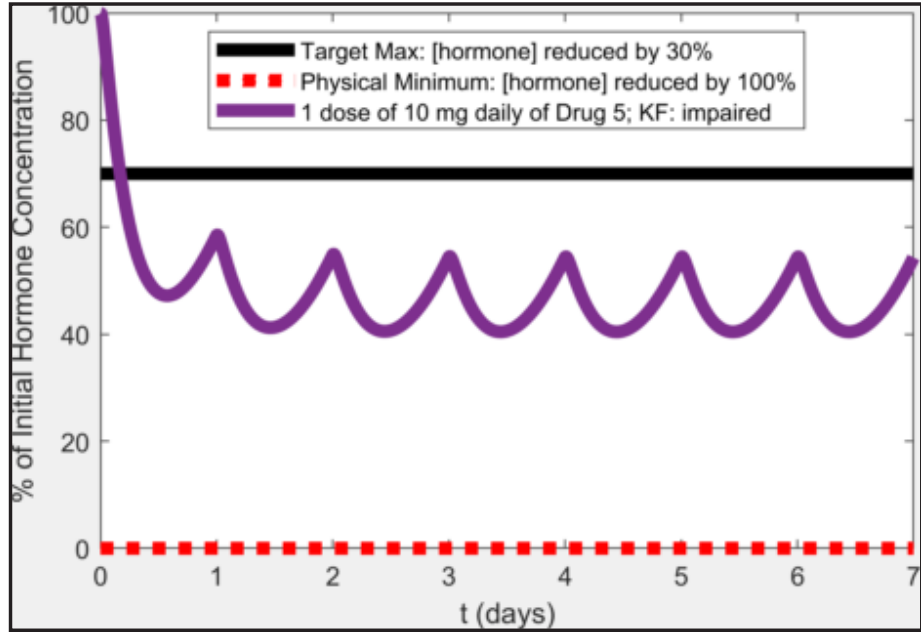

Figure 7. Possible solution for ramipril with impaired kidney function.

ing the MATLAB app on computers in a lab. Patience was needed in this step as the cohort had a wide range of computational background experience. In the second session, the children were partnered with their grandparents to complete the design for the benazepril normal KF case. When all the teams had a design they recommended, we discussed pros and cons of each design. If they finished early, ambitious older children were allowed to explore other cases for more challenge. A few of the grandparent participants simulated the results of ACE inhibitor doses for their prescription medications, which led to interesting discussions.

Another informal learning environment where we have deployed the MATLAB app is the annual Oklahoma Women in Science Conference. This event for $\sim 1000$ middle school and high school girls and their teachers featured an expo with hands-on science demonstration booths. We brought multiple laptop computers with MATLAB installed and demonstrated the MATLAB app with a student or faculty 
facilitator at each station. We invited students and teachers who attended the expo to use the app to design a drug dose amount and number of doses that lowers the hormone concentration into the therapeutic range for one case. This activity typically took 5 minutes or less, including a brief explanation by the facilitator about how chemical engineers use mathematics and knowledge of the chemical kinetics in the human body to design pharmaceutical dosages.

In the university classroom, we have used this MATLAB app in an upper division/graduate elective on scientific computing as an example to demonstrate the design and use of MATLAB apps for research and educational purposes. We recommend the use of the MATLAB app in first year engineering courses in a similar manner as used for the Summer Bridge Program, and it can be readily extended to other chemical engineering undergraduate courses such as mass and energy balances and chemical reaction kinetics. The accumulation of the drug in the digestive tract and in the circulatory system can be explained by mass balances. Each case has different parameters for the rates of absorption into the blood and elimination from the body. For chemical reaction kinetics, the scientific paper on the model details ${ }^{[5]}$ can be used to explain the reaction mechanisms behind the app. For both courses, the simulation results can be compared for different cases to interpret the relative rates of the processes involved. The parameters for each case are all contained within the mat files packaged in the code of the app.

\section{CONCLUSIONS}

The MATLAB app and design project described here are ways to work with a pharmaceutical application of chemical engineering by varying inputs and observing the results without having to do experiments. The MATLAB app is portable and can be run on any computer with the MATLAB software installed. The simulation is straightforward to run without any programming, so students spend the majority of their time running simulations and learning instead of trying to navigate the software. We recognize that MATLAB licenses are not commonly available outside of university settings. In our deployment, laptops owned by the university or by current faculty and students with active MATLAB licenses were used for external outreach events, and campus computer labs were used for university events, classes, and the Summer Bridge Program. In summary, we have provid- ed a set of resources to facilitate adoption of the MATLAB app and design project into first year engineering programs and have provided examples for other educators to consider regarding potential incorporation into additional formal and informal learning environments.

\section{ACKNOWLEDGEMENTS}

The authors would like to thank the Oklahoma State University College of Engineering, Architecture and Technology for the opportunity to develop and implement the chemical engineering design module in the CEAT Summer Bridge Program. Funding: This work was supported in part by an Award from Harold Hamm Diabetes Center at the University of Oklahoma Health Sciences Center.

\section{REFERENCES}

1. Butterfield AE, Branch K and Trujillo E (2015) First-year handson design course: implementation \& reception. Chem. Eng. Educ. 49(1):19-26

2. Harrell GK, McPeak AN and Ford Versypt AN (2017) A pharmacokinetic simulation-based module to introduce mass balances and chemical engineering design concepts to engineering freshmen. Proceedings ASEE Annual Conference, available at https://peer.asee.org/27493.

3. Ford Versypt AN, Harrell GK, McPeak AN and Eastep CE (2018) ACEInhibPKPD v2.1 Expanded GUI. http://github.com/ashleefv/ ACEInhibPKPD, http://doi.org/10.5281/zenodo.1318682.

4. Ford Versypt AN, Harrell GK and McPeak AN (2017) ACEInhibPKPD: An open-source MATLAB app for a pharmacokinetic/pharmacodynamic model of ACE inhibition. Journal of Open Source Software 2:340.

5. Ford Versypt AN, Harrell GK and McPeak AN (2017) A pharmacokinetic/pharmacodynamic model of ACE inhibition of the renin-angiotensin system for normal and impaired renal function. Computers and Chemical Engineering 104:311-322.

6. Neubeck M, Fliser D, Pritsch M, Weißer K, Fliser M, Nussberger J, Ritz E and Mutschler E (1994) Pharmacokinetics and pharmacodynamics of lisinopril in advanced renal failure. European Journal of Clinical Pharmacology 46(6):537-543.

7. Shionoiri H, Yasuda G, Ikeda A, Ohta T, Miyajima E and Kaneko Y (1987) Pharmacokinetics and depressor effect of delapril in patients with essential hypertension. Clinical Pharmacology and Therapeutics 41(1):74-79.

8. Debusmann ER, et al. (1987) Influence of renal function on the pharmacokinetics of ramipril (HOE 498). American Journal of Cardiology 59(10):D70-D78

9. Ocon-Pujadas J, Debusmann ER, Jane F, Lahn W, Irmisch R, Mora $\mathrm{J}$ and Grotsch H (1989) Pharmacodynamic effects of a single 10-mg dose of the angiotensin converting enzyme inhibitor ramipril in patients with impaired renal function. Journal of Cardiovascular Pharmacology 13:S45-S48

10. TeachEngineering, Engineering Design Process https://www.teachengineering.org/k12engineering/designprocess accessed February 9, 2019 